\title{
Convergence Analysis of The Finite Difference Solution for Two Dimensions Coupled-Benjamin-Bona-Mahony System
}

Ekhlass S. Al-Rawi

drekhlass-alrawi@uomosul.edu.iq

College of Computer Sciences and Mathematics

University of Mosul, Mosul, Iraq

Received on: 20/05/2012

\section{Muhannad Shallal}

College of Science

University of Kirkuk, Kirkuk, Iraq

Accepted on: 18/09/2012

\section{ABSTRACT}

This paper is devoted to drive the matrix algebraic equation for the twodimensional nonlinear coupled-BBM system which is obtained from using the implicit finite difference method. The convergence analysis of the solution is proved. Numerical experiment is presented with initial conditions describing the generation and evolution.

Keywords: Boussinesq system; Solitary waves ; Implicit finite difference method; Convergence analysis.
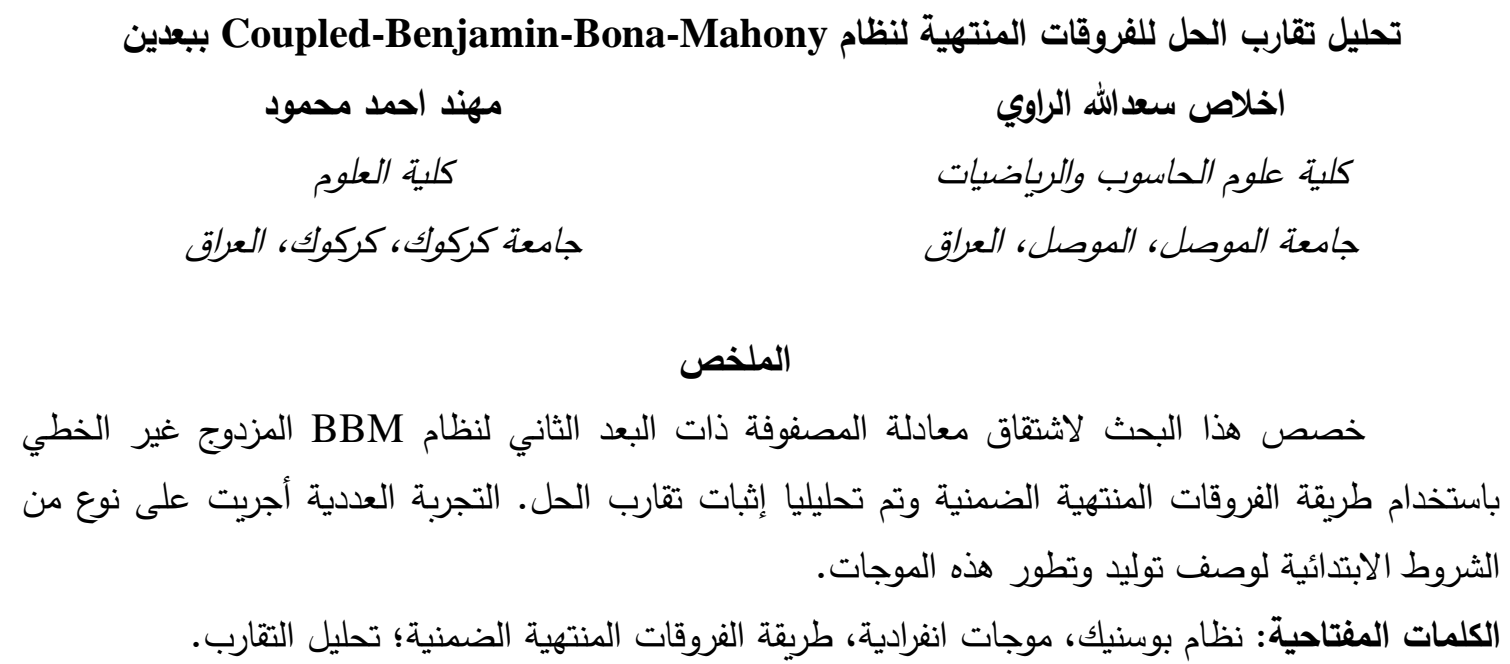

1. Introduction.

In recent years, there have been many theoretical and computational methods in the study of the full water-wave problem. However, there is still need for accurate, simpler mathematical models. Boussinesq systems of partial differential equations that approximate the three dimensional Euler equations that describe the irrotational, free surface flow of an incompressible, inviscid fluid. Because of their simplicity, Boussinesq systems have been used in the study of a varity of water wave phenomena in ports, channels, coastal areas, and in the open sea. They have also been used in studies of tsunami wave generation and propagation [16]. These systems may be written as follows:

$$
\begin{aligned}
& v_{t}+\nabla u+\nabla(v u)+a \Delta \nabla u-b \Delta v_{t}=0 \\
& u_{t}+\nabla v+\frac{1}{2} \nabla|u|^{2}+c \Delta \nabla v-d \Delta u_{t}=0 .
\end{aligned}
$$


These systems have been derived by[9] as approximations to the threedimensional Euler equations and describe irrotational free surface flow of an ideal fluid over a horizontal bottom. The independent variable $X=(x, y)$ represents the position, $\mathrm{t}$ is proportional to elapsed time, $v=v(X, t)$ is proportional to the deviation of the free surface from its rest position, while $\mathrm{u}$ is proportional to the horizontal velocity of the fluid at some height. We would also like to mention that the initial-boundary value problem for the analogous BBM-BBM system in one space dimension with nonhomogeneous Dirichlet boundary conditions at the endpoints of a finite interval were shown to be locally well-posed by Bona and Chen, [7]. Initial-boundary-value problems (ibvp's) for (1) on a finite interval in one space variable were analyzed in [6]. In [3] we proved the convergence analysis of the solution of one-dimensional nonlinear Coupled-BBM system by using implicit finite difference method. In [17] the authors proved optimal-order L2-error estimates for the standared Galerkin semidiscretization of the BBM-BBM system with homogeneous boundary conditions on a smooth domain with a general triangulation. Three ibvp's for the BBM-BBM system on a smooth plane domain, corresponding to homogeneous Dirichlet boundary conditions, homogeneous Neumann boundary conditions and to the (normal) reflective boundary conditions analyzed in [18]. Solution for one dimensional Benjamin-Bona-Mahony-Burgers equation using Crank-Nicolson Finite Difference method introduced in [10 ], while Huang in [15 ] solved Coupled BBM- system in one dimension by using homotopy analysis method. Existence of the solitary waves for these, one-dimensional system has been studied by Toland [5]. The wellposedness and regularity of (1) are established in [7 ] and [ 8]. The existence of line solitary waves, line cnoidal waves, symmetric and a symmetric periodic wave patterns are proved in [4, 11, 12 ]and [13].

In this paper, we will study the Boussinesq system of the type BBM-BBM

corresponds to $a=c=0, b=d=1 / 6$, i.e

$v_{t}+\nabla u+\nabla(v u)-\frac{1}{6} \Delta v_{t}=0$

$u_{t}+\nabla v+\frac{1}{2} \nabla|u|^{2}-\frac{1}{6} \Delta u_{t}=0$

where $v, u$ are mappings from $\Omega \times(0, \infty)$ to $\Re, \Omega=(0, L) \times(0, L)$ with boundary conditions: $\left.v\right|_{\partial \Omega}=0,\left.u\right|_{\partial \Omega}=0, t \in[0, \infty)$ and the initial conditions are $\left.v\right|_{t=0}=v_{0},\left.u\right|_{t=0}=u_{0}, X \in \Omega$. [14]

\section{Derivation of the Matrix Equation Using the Finite Difference Method.}

Divide the interval $(0, \mathrm{~L})$ in the $\mathrm{x}$ direction into $\mathrm{n}$ subintervals $\left(x_{k-1}, x_{k}\right)$ of the uniform length $\frac{1}{n}, k=1, \ldots, n, 0=x_{0}\left\langle x_{1}\left\langle\ldots\left\langle x_{n-1}\left\langle x_{n}=L\right.\right.\right.\right.$, and divide the interval $(0, \mathrm{~L})$ in the $y$ direction into the same number of subintervals $\left(y_{k-1}, y_{k}\right)$ of the uniform length $\frac{1}{n}, k=1, \ldots, n, 0=y_{0}\left\langle y_{1}<\ldots<y_{n-1}<y_{n}=L\right.$.

The discredited solution of the equation (2a) and multiply equation by $\mathrm{k}$, we get the following equation 


$$
\begin{aligned}
& \left(v^{j}\left(x_{k}, y_{s}\right)-v^{j-1}\left(x_{k}, y_{s}\right)\right)+\frac{k}{2 h}\left(u^{j-1}\left(x_{k+1}, y_{s}\right)-u^{j-1}\left(x_{k-1}, y_{s}\right)\right)+\frac{k}{2 h}\left(u^{j-1}\left(x_{k}, y_{s+1}\right)-u^{j-1}\left(x_{k}, y_{s-1}\right)\right)+ \\
& \frac{k v^{j}\left(x_{k}, y_{s}\right)}{2 h}\left(u^{j}\left(x_{k+1}, y_{s}\right)-u^{j}\left(x_{k-1}, y_{s}\right)\right)+\frac{k u^{j}\left(x_{k}, y_{s}\right)}{2 h}\left(v^{j}\left(x_{k+1}, y_{s}\right)-v^{j}\left(x_{k-1}, y_{s}\right)\right)+ \\
& \frac{k v^{j}\left(x_{k}, y_{s}\right)}{2 h}\left(u^{j}\left(x_{k}, y_{s+1}\right)-u^{j}\left(x_{k}, y_{s-1}\right)\right)+\frac{k u^{j}\left(x_{k}, y_{s}\right)}{2 h}\left(v^{j}\left(x_{k}, y_{s+1}\right)-v^{j}\left(x_{k}, y_{s-1}\right)\right) \\
& -\frac{1}{6 h^{2}}\left(v^{j}\left(x_{k+1}, y_{s}\right)-2 v^{j}\left(x_{k}, y_{s}\right)+v^{j}\left(x_{k-1}, y_{s}\right)-v^{j-1}\left(x_{k+1}, y_{s}\right)+2 v^{j-1}\left(x_{k}, y_{s}\right)-v^{j-1}\left(x_{k-1}, y_{s}\right)\right) \\
& -\frac{1}{6 h^{2}}\left(v^{j}\left(x_{k}, y_{s+1}\right)-2 v^{j}\left(x_{k}, y_{s}\right)+v^{j}\left(x_{k}, y_{s-1}\right)-v^{j-1}\left(x_{k}, y_{s+1}\right)+2 v^{j-1}\left(x_{k}, y_{s}\right)-v^{j-1}\left(x_{k}, y_{s-1}\right)\right)=0,
\end{aligned}
$$

$k, s=1, \ldots, n-1$.

After arranging the above equation in terms of $v^{j}\left(x_{k-1}, y_{s}\right), v^{j}\left(x_{k}, y_{s}\right), v^{j}\left(x_{k+1}, y_{s}\right), v^{j}\left(x_{k}, y_{s-1}\right)$ and $v^{j}\left(x_{k}, y_{s+1}\right)$, we get

$G_{2}\left(u^{j}\left(x_{k}, y_{s}\right)\right) v^{j}\left(x_{k-1}, y_{s}\right)+G_{3}\left(u^{j}\left(x_{k}, y_{s}\right)\right) v^{j}\left(x_{k+1}, y_{s}\right)+G_{1}\left(u^{j}\left(x_{k}, y_{s}\right)\right) v^{j}\left(x_{k}, y_{s}\right)+$ $G_{2}\left(u^{j}\left(x_{k}, y_{s}\right)\right) v^{j}\left(x_{k}, y_{s-1}\right)+G_{3}\left(u^{j}\left(x_{k}, y_{s}\right)\right) v^{j}\left(x_{k}, y_{s+1}\right)=H\left(u^{j-1}\left(x_{k}, y_{s}\right), v^{j-1}\left(x_{k}, y_{s}\right)\right)$, (3) where

$$
\begin{aligned}
& G_{1}\left(u^{j}\left(x_{k}, y_{s}\right)\right)=\left(1+\frac{k}{2 h}\left(u^{j}\left(x_{k+1}, y_{s}\right)-u^{j}\left(x_{k-1}, y_{s}\right)+u^{j}\left(x_{k}, y_{s+1}\right)-u^{j}\left(x_{k}, y_{s-1}\right)\right)+\frac{2}{3 h^{2}}\right), \\
& G_{2}\left(u^{j}\left(x_{k}, y_{s}\right)\right)=\left(-\frac{k}{2 h} u^{j}\left(x_{k}, y_{s}\right)-\frac{1}{6 h^{2}}\right), \\
& G_{3}\left(u^{j}\left(x_{k}, y_{s}\right)\right)=\left(\frac{k}{2 h} u^{j}\left(x_{k}, y_{s}\right)-\frac{1}{6 h^{2}}\right),
\end{aligned}
$$

and

$$
\begin{aligned}
& H\left(u^{j-1}\left(x_{k}, y_{s}\right), v^{j-1}\left(x_{k}, y_{s}\right)\right)=v^{j-1}\left(x_{k}, y_{s}\right)-\frac{k}{2 h}\left(u^{j-1}\left(x_{k+1}, y_{s}\right)-u^{j-1}\left(x_{k-1}, y_{s}\right)\right) \\
& -\frac{k}{2 h}\left(u^{j-1}\left(x_{k}, y_{s+1}\right)-u^{j-1}\left(x_{k}, y_{s-1}\right)\right)-\frac{1}{6 h^{2}} v^{j-1}\left(x_{k+1}, y_{s}\right)+\frac{2}{3 h^{2}} v^{j-1}\left(x_{k}, y_{s}\right) \\
& -\frac{1}{6 h^{2}} v^{j-1}\left(x_{k-1}, y_{s}\right)-\frac{1}{6 h^{2}} v^{j-1}\left(x_{k}, y_{s+1}\right)-\frac{1}{6 h^{2}} v^{j-1}\left(x_{k}, y_{s-1}\right) .
\end{aligned}
$$

Equation (3) becomes the following matrix equation $G\left(u^{j}, v^{j}\right)\left\{v^{j}\right\}=\left\{H\left(u^{j-1}, v^{j-1}\right)\right\}$, where $G$ is $(n-1)^{2} \times(n-1)^{2}$ matrix and can be represented by the matrix of $(n-1) \times(n-1)$ submatrices $g_{l, s}\left(u^{j}, v^{j}\right)$ for $l, s=1,2, \ldots, n-1$, that is

$$
\left[\begin{array}{ccc}
g_{1,1}\left(u^{j}, v^{j}\right) & \cdots & g_{1, n-1}\left(u^{j}, v^{j}\right) \\
g_{2,1}\left(u^{j}, v^{j}\right) & \cdots & g_{2, n-1}\left(u^{j}, v^{j}\right) \\
\vdots & \vdots & \vdots \\
g_{n-2,1}\left(u^{j}, v^{j}\right) & \cdots & g_{n-2, n-1}\left(u^{j}, v^{j}\right) \\
g_{n-1,1}\left(u^{j}, v^{j}\right) & \vdots & g_{n-1, n-1}\left(u^{j}, v^{j}\right)
\end{array}\right] .
$$

The main diagonal entries $g_{l, l}, l=1, \ldots, n-1$ and other diagonal entries $g_{l, l-1}, g_{l-1, l}, l=2, \ldots, n-1$ are the only nonzero elements. we denote the nonzero elements of $g_{l, l}, g_{l, l-1}$ and $g_{l-1, l}$ by $\left(g_{i}^{j}\right)_{k(l), s(l)}=g_{i}\left(u^{j}\left(x_{k(l)}, y_{s(l)}\right)\right), i=1,2,3$. 
Then the $g_{l, l}\left(u^{j}, v^{j}\right), l=1, \ldots, n-1$ is the following tri-diagonal matrix: $\left[\begin{array}{ccccc}\left(g_{1}^{j}\right)_{(l-1) n-(l-2), 1} & g_{3} & 0 & 0 & 0 \\ g_{2} & \left(g_{1}^{j}\right)_{(l-1) n-(l-3), 2} & g_{3} & 0 & 0 \\ \vdots & \vdots & \ddots & \vdots & \vdots \\ 0 & 0 & g_{2} & \left(g_{1}^{j}\right)_{l(n-1)-1, n-2} & g_{3} \\ 0 & 0 & 0 & g_{2} & \left(g_{1}^{j}\right)_{l(n-1), n-1}\end{array}\right]$.

The two other diagonal elements $g_{l, l-1}, g_{l-1, l}, l=2, \ldots, n-1$ are the following diagonal matrices respectively:

$\left[\begin{array}{ccccc}\left(g_{2}^{j}\right)_{(l-1) n-(l-2), 1} & 0 & 0 & 0 & 0 \\ 0 & \left(g_{2}^{j}\right)_{(l-1) n-(l-3), 2} & 0 & 0 & 0 \\ \vdots & \vdots & \ddots & \vdots & \vdots \\ 0 & 0 & 0 & \left(g_{2}^{j}\right)_{l(n-1)-1, n-2} & 0 \\ 0 & 0 & 0 & 0 & \left(g_{2}^{j}\right)_{l(n-1), n-1}\end{array}\right]$

and

$$
\left[\begin{array}{ccccc}
\left(g_{3}^{j}\right)_{(l-2) n-(l-3), 1} & 0 & 0 & 0 & 0 \\
0 & \left(g_{3}^{j}\right)_{(l-2) n-(l-4), 2} & 0 & 0 & 0 \\
\vdots & \vdots & \ddots & \vdots & \vdots \\
0 & 0 & 0 & \left(g_{3}^{j}\right)_{(l-1)(n-1)-1, n-2} & 0 \\
0 & 0 & 0 & 0 & \left(g_{3}^{j}\right)_{(l-1)(n-1), n-1}
\end{array}\right] .
$$

The $\left\{v^{j}\right\}$ is a $(n-1)^{2} \times 1$ vector and it is also considered as $(n-1) \times 1$ vector of $(\mathrm{n}-1)$ subvector $\left\{v_{i}^{j}\right\}, i=1, \ldots, n-1$. That is

$\left\{v^{j}\right\}=\left\{\left\{v_{1}^{j}\right\},\left\{v_{2}^{j}\right\}, \ldots,\left\{v_{n-1}^{j}\right\}\right\}^{T}$,

where

$\left\{v_{i}^{j}\right\}=\left\{v^{j}\left(x_{i}, y_{1}\right), \ldots, v^{j}\left(x_{i}, y_{n-1}\right)\right\}^{T}$.

Similarly

$\left\{H\left(u^{j-1}, v^{j-1}\right)\right\}=\left\{\left\{H_{1}^{j}\right\}, \ldots,\left\{H_{n-1}^{j}\right\}\right\}$,

where

$\left\{H_{i}^{j}\right\}=\left\{H^{j}\left(x_{i}, y_{1}\right), \ldots, H^{j}\left(x_{i}, y_{n-1}\right)\right\}^{T}$.

By the same approach for the equation(2b), we get

$P_{2}\left(u^{j}\left(x_{k}, y_{s}\right)\right) u^{j}\left(x_{k-1}, y_{s}\right)+P_{2}\left(u^{j}\left(x_{k}, y_{s}\right)\right) u^{j}\left(x_{k+1}, y_{s}\right)+P_{1}\left(u^{j}\left(x_{k}, y_{s}\right)\right) u^{j}\left(x_{k}, y_{s}\right)+$

$P_{2}\left(u^{j}\left(x_{k}, y_{s}\right)\right) u^{j}\left(x_{k}, y_{s-1}\right)+P_{2}\left(u^{j}\left(x_{k}, y_{s}\right)\right) u^{j}\left(x_{k}, y_{s+1}\right)=Q\left(u^{j-1}\left(x_{k}, y_{s}\right), v^{j-1}\left(x_{k}, y_{s}\right)\right)$

Where,

$$
\begin{aligned}
& P_{1}\left(u^{j}\left(x_{k}, y_{s}\right)\right)=\left(1+\frac{k}{2 h}\left(u^{j}\left(x_{k+1}, y_{s}\right)-u^{j}\left(x_{k-1}, y_{s}\right)+u^{j}\left(x_{k}, y_{s+1}\right)-u^{j}\left(x_{k}, y_{s-1}\right)\right)+\frac{2}{3 h^{2}}\right) \\
& P_{2}\left(u^{j}\left(x_{k}, y_{s}\right)\right)=-\frac{1}{6 h^{2}},
\end{aligned}
$$

and 


$$
\begin{aligned}
& Q\left(u^{j-1}\left(x_{k}, y_{s}\right), v^{j-1}\left(x_{k}, y_{s}\right)\right)=u^{j-1}\left(x_{k}, y_{s}\right)-\frac{k}{2 h}\left(v^{j-1}\left(x_{k+1}, y_{s}\right)-v^{j-1}\left(x_{k-1}, y_{s}\right)\right) \\
& -\frac{k}{2 h}\left(v^{j-1}\left(x_{k}, y_{s+1}\right)-v^{j-1}\left(x_{k}, y_{s-1}\right)\right)-\frac{1}{6 h^{2}} u^{j-1}\left(x_{k+1}, y_{s}\right)+\frac{2}{3 h^{2}} u^{j-1}\left(x_{k}, y_{s}\right) \\
& -\frac{1}{6 h^{2}} u^{j-1}\left(x_{k-1}, y_{s}\right)-\frac{1}{6 h^{2}} u^{j-1}\left(x_{k}, y_{s+1}\right)-\frac{1}{6 h^{2}} u^{j-1}\left(x_{k}, y_{s-1}\right) .
\end{aligned}
$$

Equation (4) becomes the following matrix equation $P\left(u^{j}, v^{j}\right)\left\{u^{j}\right\}=\left\{Q\left(u^{j-1}, v^{j-1}\right\}\right.$,

Where, $P\left(u^{j}, v^{j}\right)$ is $(n-1)^{2} \times(n-1)^{2}$ matrix and can be represented by the matrix of $(n-1) \times(n-1)$ submatrices $p_{l, s}\left(u^{j}, v^{j}\right)$ for $l, s=1,2, \ldots, n-1$, that is

$$
\left[\begin{array}{ccc}
p_{1,1}\left(u^{j}, v^{j}\right) & \cdots & p_{1, n-1}\left(u^{j}, v^{j}\right) \\
p_{2,1}\left(u^{j}, v^{j}\right) & \cdots & p_{2, n-1}\left(u^{j}, v^{j}\right) \\
\vdots & \vdots & \vdots \\
p_{n-2,1}\left(u^{j}, v^{j}\right) & \cdots & p_{n-2, n-1}\left(u^{j}, v^{j}\right) \\
p_{n-1,1}\left(u^{j}, v^{j}\right) & \vdots & p_{n-1, n-1}\left(u^{j}, v^{j}\right)
\end{array}\right] .
$$

The main diagonal entries $p_{l, l}\left(u^{j} v^{j}\right), l=1, \ldots, n-1$ and other diagonal entries $p_{l, l-1}\left(u^{j}, v^{j}\right), p_{l-1, l}\left(u^{j}, v^{j}\right), l=2, \ldots, n-1$ are the only nonzero elements. We denote $p_{i}\left(u^{j}\left(x_{k(l)}, y_{s(l)}\right), v^{j}\left(x_{k(l)}, y_{s(l)}\right)\right)$ the nonzero elements of $p_{l, l}\left(u^{j}, v^{j}\right), p_{l, l-1}\left(u^{j}, v^{j}\right)$ or $p_{l-1, l}\left(u^{j}, v^{j}\right)$ by $\left(p_{i}^{j}\right)_{k(l), s(l)}, i=1,2$. Then, the $p_{l, l}\left(u^{j}, v^{j}\right), l=1, \ldots, n-1$ is the following tri-diagonal matrix:

$$
\left[\begin{array}{ccccc}
\left(p_{1}^{j}\right)_{(l-1) n-(l-2), 1} & p_{2} & 0 & 0 & 0 \\
p_{2} & \left(p_{1}^{j}\right)_{(l-1) n-(l-3), 2} & p_{2} & 0 & 0 \\
\vdots & \vdots & \ddots & \vdots & \vdots \\
0 & 0 & p_{2} & \left(p_{1}^{j}\right)_{l(n-1)-1, n-2} & p_{2} \\
0 & 0 & 0 & p_{2} & \left(p_{1}^{j}\right)_{l(n-1), n-1}
\end{array}\right] .
$$

The two other diagonal elements $p_{l, l-1}\left(u^{j}, v^{j}\right), p_{l-1, l}\left(u^{j}, v^{j}\right), l=2, \ldots, n-1$ are the following diagonal matrices :

$$
\left[\begin{array}{ccccc}
p_{2} & 0 & 0 & 0 & 0 \\
0 & p_{2} & 0 & 0 & 0 \\
\vdots & \vdots & \ddots & \vdots & \vdots \\
0 & 0 & 0 & p_{2} & 0 \\
0 & 0 & 0 & 0 & p_{2}
\end{array}\right]
$$

Where, $\left\{u^{j}\right\},\left\{H\left(u^{j-1}, v^{j-1}\right)\right\}$ are $(n-1)^{2} \times 1$ vector.

We now prove the existence of the solutions of the matrices equations which we obtained from equation (3) and equation (4) respectively.

Theorem 2.1. The solutions of the matrices equations

$$
\text { (i) } G\left(u^{j}, v^{j}\right)\left\{v^{j}\right\}=\left\{H\left(u^{j-1}, v^{j-1}\right)\right\}
$$

and 
exist.

(ii) $P\left(u^{j}, v^{j}\right)\left\{u^{j}\right\}=\left\{Q\left(u^{j-1}, v^{j-1}\right)\right\}$

Proof (i): let $j \in N$ be fixed, consider the following iteration

$G\left(u_{i-1}^{j}\right)\left\{v_{i}^{j}\right\}=\left\{H\left(u^{j-1}, v^{j-1}\right)\right\}$, where $u_{0}^{j}=u^{j-1}$. By subtracting the last equation from $G\left(u_{i}^{j}\right)\left\{v_{i+1}^{j}\right\}=\left\{H\left(u^{j-1}, v^{j-1}\right)\right\}$, we get

$G\left(u_{i}^{j}\right)\left\{v_{i+1}^{j}\right\}-G\left(u_{i-1}^{j}\right)\left\{v_{i}^{j}\right\}=0$, then

$G\left(u_{i}^{j}, v_{i}^{j}\right)\left\{v_{i+1}^{j}-v_{i}^{j}\right\}=\left(G\left(u_{i-1}^{j}\right)-G\left(u_{i}^{j}, v_{i}^{j}\right)\right)\left\{v_{i}^{j}\right\}$.

For the first row in the right hand side of last equation

$\left(G_{1}\left(u_{i-1}\left(x_{1}, y_{1}\right)\right)-G_{1}\left(u_{i}\left(x_{1}, y_{1}\right)\right)\right) v_{i}^{j}\left(x_{1}, y_{1}\right)+\left(G_{3}\left(u_{i-1}\left(x_{1}, y_{1}\right)\right)-G_{3}\left(u_{i}\left(x_{1}, y_{1}\right)\right)\right) v_{i}^{j}\left(x_{1}, y_{2}\right)$

$+\left(G_{3}\left(u_{i-1}\left(x_{1}, y_{1}\right)\right)-G_{3}\left(u_{i}\left(x_{1}, y_{1}\right)\right)\right) v_{i}^{j}\left(x_{2}, y_{1}\right)$.

By mean value theorem, the first row elements become

$\delta_{1, l}^{j}=\sum_{l=1}^{n-1}\left[\left[G_{1}\left(u_{i^{*}}^{j}\left(x_{1}, y_{1}\right)\right)\right]_{x l} v_{i}^{j}\left(x_{1}, y_{1}\right)+\left[G_{3}\left(u_{i^{*}}^{j}\left(x_{1}, y_{1}\right)\right)\right]_{x l} v_{i}^{j}\left(x_{1}, y_{2}\right)\right.$

$\left.+\left[G_{3}\left(u_{i^{*}}^{j}\left(x_{1}, y_{1}\right)\right)\right]_{x l} v_{i}^{j}\left(x_{2}, y_{1}\right)\right]\left(u_{i-1}\left(x_{1}, y_{1}\right)\right)-\left(u_{i}\left(x_{1}, y_{1}\right)\right)$,

where the value of $u_{i^{*}}^{j}\left(x_{1}, y_{1}\right)$ is between $u_{i-1}^{j}\left(x_{1}, y_{1}\right)$ and $u_{i}^{j}\left(x_{1}, y_{1}\right)$, and $\left(G_{i}\left(u_{i^{*}}^{j}\left(x_{1}, y_{1}\right)\right)\right)_{x_{l}}, i=1,3$ represents the partial derivatives of $\left(G_{i}\left(u_{i^{*}}^{j}\left(x_{1}, y_{1}\right)\right)\right)$ with respect to $u_{i^{*}}^{j}\left(x_{l}, y_{1}\right)$.

Now for the first element

$$
\begin{gathered}
\delta_{1,1}^{j}=\left[G_{1}\left(u_{i^{*}}^{j}\left(x_{1}, y_{1}\right)\right)\right]_{x 1} v_{i}^{j}\left(x_{1}, y_{1}\right)\left[G_{3}\left(u_{i^{*}}^{j}\left(x_{1}, y_{1}\right)\right)\right]_{x 1} v_{i}^{j}\left(x_{1}, y_{2}\right)+ \\
\left.+\left[G_{3}\left(u_{i^{*}}^{j}\left(x_{1}, y_{1}\right)\right)\right]_{x 1} v_{i}^{j}\left(x_{2}, y_{1}\right)\right]\left(u_{i-1}\left(x_{1}, y_{1}\right)\right)-\left(u_{i}\left(x_{1}, y_{1}\right)\right) \\
=\left(0+\frac{k}{2 h} v_{i}^{j}\left(x_{1}, y_{2}\right)+\frac{k}{2 h} v_{i}^{j}\left(x_{2}, y_{1}\right)\right)\left(u_{i-1}\left(x_{1}, y_{1}\right)\right)-\left(u_{i}\left(x_{1}, y_{1}\right)\right), \\
\delta_{1,2}^{j}=\left[G_{1}\left(u_{i^{*}}^{j}\left(x_{1}, y_{1}\right)\right)\right]_{x 2} v_{i}^{j}\left(x_{1}, y_{1}\right)\left[G_{3}\left(u_{i^{*}}^{j}\left(x_{1}, y_{1}\right)\right)\right]_{x 2} v_{i}^{j}\left(x_{1}, y_{2}\right)+ \\
\left.+\left[G_{3}\left(u_{i^{*}}^{j}\left(x_{1}, y_{1}\right)\right)\right]_{x 2} v_{i}^{j}\left(x_{2}, y_{1}\right)\right]\left(u_{i-1}\left(x_{1}, y_{1}\right)\right)-\left(u_{i}\left(x_{1}, y_{1}\right)\right) \\
=\left(\frac{k}{2 h} v_{i}^{j}\left(x_{1}, y_{1}\right)\right)\left(u_{i-1}\left(x_{1}, y_{1}\right)\right)-\left(u_{i}\left(x_{1}, y_{1}\right)\right) .
\end{gathered}
$$

For the second row, we have

$$
\begin{aligned}
& \delta_{2, l}^{j}=\sum_{l=1}^{n-1}\left[\left[G_{2}\left(u_{i^{*}}^{j}\left(x_{1}, y_{2}\right)\right)\right]_{x l} v_{i}^{j}\left(x_{1}, y_{1}\right)+\left[G_{1}\left(u_{i^{*}}^{j}\left(x_{1}, y_{2}\right)\right)\right]_{x l} v_{i}^{j}\left(x_{1}, y_{2}\right)\right. \\
& \left.\left.+\left[G_{3}\left(u_{i^{*}}^{j}\left(x_{1}, y_{2}\right)\right)\right]_{x l} v_{i}^{j}\left(x_{1}, y_{3}\right)+G_{3}\left(u_{i^{*}}^{j}\left(x_{1}, y_{2}\right)\right)\right]_{x l} v_{i}^{j}\left(x_{2}, y_{2}\right)\right]\left(u_{i-1}\left(x_{1}, y_{2}\right)\right)-G_{1}\left(u_{i}\left(x_{1}, y_{2}\right)\right), \\
& \quad l=1,2, \ldots, n-1 .
\end{aligned}
$$

the first element in the second row, becomes

$$
\begin{aligned}
& \delta_{2,1}^{j}=\left[G_{2}\left(u_{i^{*}}^{j}\left(x_{1}, y_{2}\right)\right)\right]_{x 1} v_{i}^{j}\left(x_{1}, y_{1}\right)+\left[G_{1}\left(u_{i^{*}}^{j}\left(x_{1}, y_{2}\right)\right)\right]_{x 1} v_{i}^{j}\left(x_{1}, y_{2}\right) \\
& \left.\left.+\left[G_{3}\left(u_{i^{*}}^{j}\left(x_{1}, y_{2}\right)\right)\right]_{x 1} v_{i}^{j}\left(x_{1}, y_{3}\right)+G_{3}\left(u_{i^{*}}^{j}\left(x_{1}, y_{2}\right)\right)\right]_{x 1} v_{i}^{j}\left(x_{2}, y_{2}\right)\right]\left(u_{i-1}\left(x_{1}, y_{2}\right)\right)-G_{1}\left(u_{i}\left(x_{1}, y_{2}\right)\right) \\
& =\left(-\frac{k}{2 h} v_{i}^{j}\left(x_{1}, y_{1}\right)+0+\frac{k}{2 h} v_{i}^{j}\left(x_{1}, y_{3}\right)+\frac{k}{2 h} v_{i}^{j}\left(x_{2}, y_{2}\right)\right)\left(u_{i-1}\left(x_{1}, y_{2}\right)\right)-G_{1}\left(u_{i}\left(x_{1}, y_{2}\right)\right),
\end{aligned}
$$




$$
\begin{aligned}
& \delta_{(n-1)^{2}-1,(n-1)^{2}}^{j}=\left[\left[G_{2}\left(u_{i^{*}}^{j}\left(x_{n-1}, y_{n-1}\right)\right)\right]_{x_{n-2}} v_{i}^{j}\left(x_{n-2}, y_{n-1}\right)+\left[G_{2}\left(u_{i^{*}}^{j}\left(x_{n-1}, y_{n-1}\right)\right)\right]_{x_{n-2}} v_{i}^{j}\left(x_{n-1}, y_{n-2}\right)\right. \\
& \left.+\left[G_{1}\left(u_{i^{*}}^{j}\left(x_{n-1}, y_{n-1}\right)\right)\right]_{x_{n-2}} v_{i}^{j}\left(x_{n-1}, y_{n-1}\right)\right]\left(u_{i-1}\left(x_{n-1}, y_{n-1}\right)\right)-G_{1}\left(u_{i}\left(x_{n-1}, y_{n-1}\right)\right) \\
& \quad=\left(0+0+\frac{-k}{2 h} v_{i}^{j}\left(x_{n-1}, y_{n-1}\right)\right)\left(u_{i-1}\left(x_{n-1}, y_{n-1}\right)\right)-\left(u_{i}\left(x_{n-1}, y_{n-1}\right)\right),
\end{aligned}
$$

and

$$
\begin{aligned}
& \delta_{(n-1)^{2},(n-1)^{2}-1}^{j}= {\left[\left[G_{2}\left(u_{i^{*}}^{j}\left(x_{n-1}, y_{n-2}\right)\right)\right]_{x_{n-1}} v_{i}^{j}\left(x_{n-2}, y_{n-2}\right)+\left[G_{2}\left(u_{i^{*}}^{j}\left(x_{n-1}, y_{n-2}\right)\right)\right]_{x_{n-1}} v_{i}^{j}\left(x_{n-1}, y_{n-3}\right)\right.} \\
&\left.\left.+\left[G_{1}\left(u_{i^{*}}^{j}\left(x_{n-1}, y_{n-2}\right)\right)\right]_{x_{n-1}} v_{i}^{j}\left(x_{n-1}, y_{n-2}\right)\right]+\left[G_{3}\left(u_{i^{*}}^{j}\left(x_{n-1}, y_{n-2}\right)\right)\right]_{x_{n-1}} v_{i}^{j}\left(x_{n-1}, y_{n-1}\right)\right]^{*} \\
&\left(u_{i-1}\left(x_{n-1}, y_{n-2}\right)\right)-\left(u_{i}\left(x_{n-1}, y_{n-2}\right)\right) \\
&=\left(\frac{-k}{2 h} v_{i}^{j}\left(x_{n-2}, y_{n-2}\right)+\frac{-k}{2 h} v_{i}^{j}\left(x_{n-1}, y_{n-3}\right)+\frac{k}{2 h} v_{i}^{j}\left(x_{n-1}, y_{n-1}\right)\right)\left(u_{i-1}\left(x_{n-1}, y_{n-2}\right)\right)-\left(u_{i}\left(x_{n-1}, y_{n-2}\right)\right) .
\end{aligned}
$$

Therefore, the right hand side of equation (5) becomes

$$
R\left(v_{i}^{j}, u_{i^{*}}^{j}\right)\left\{u_{i-1}^{j}-u_{i}^{j}\right\} \text {, }
$$

where $R\left(v_{i}^{j}, u_{i^{*}}^{j}\right)$ is the following matrix

$$
\left[\begin{array}{ccccc}
r_{1,1}^{j} & r_{1,2}^{j} & 0 & 0 & 0 \\
r_{2,1}^{j} & r_{2,2}^{j} & r_{2,3}^{j} & 0 & 0 \\
\vdots & \vdots & \ddots & \vdots & \vdots \\
0 & 0 & r_{(n-1)^{2}-1,(n-1)^{2}-2}^{j} & r_{(n-1)^{2}-1,(n-1)^{2}-1}^{j} & r_{(n-1)^{2}-1,(n-1)^{2}}^{j} \\
0 & 0 & 0 & r_{(n-1)^{2},(n-1)^{2}-1} & r_{(n-1)^{2},(n-1)^{2}}^{j}
\end{array}\right] .
$$

Since, $\mathrm{k}$ and $\mathrm{h}$ are both small and $v^{j}$ is bounded [1], then the nonzero elements are sufficiently small.

Since,

$$
\begin{aligned}
r_{1,1}^{j}= & {\left[G_{1}\left(u_{i^{*}}^{j}\left(x_{1}, y_{1}\right)\right)\right]_{x 1} v_{i}^{j}\left(x_{1}, y_{1}\right)\left[G_{3}\left(u_{i^{*}}^{j}\left(x_{1}, y_{1}\right)\right)\right]_{x 1} v_{i}^{j}\left(x_{1}, y_{2}\right)+} \\
& \left.+\left[G_{3}\left(u_{i^{*}}^{j}\left(x_{1}, y_{1}\right)\right)\right]_{x 1} v_{i}^{j}\left(x_{2}, y_{1}\right)\right]\left(u_{i-1}\left(x_{1}, y_{1}\right)\right)-\left(u_{i}\left(x_{1}, y_{1}\right)\right) \\
= & 0+\frac{k}{2 h} v_{i}^{j}\left(x_{1}, y_{2}\right)+\frac{k}{2 h} v_{i}^{j}\left(x_{2}, y_{1}\right) \\
\leq & \frac{k}{2 h}\left(\left|v_{i}^{j}\left(x_{1}, y_{2}\right)\right|+\left|v_{i}^{j}\left(x_{2}, y_{1}\right)\right|\right) \leq \epsilon_{1}, \\
r_{1,2}^{j}= & {\left[G_{1}\left(u_{i^{*}}^{j}\left(x_{1}, y_{1}\right)\right)\right]_{x 2} v_{i}^{j}\left(x_{1}, y_{1}\right)\left[G_{3}\left(u_{i^{*}}^{j}\left(x_{1}, y_{1}\right)\right)\right]_{x 2} v_{i}^{j}\left(x_{1}, y_{2}\right)+} \\
& \left.\quad+\left[G_{3}\left(u_{i^{*}}^{j}\left(x_{1}, y_{1}\right)\right)\right]_{x 2} v_{i}^{j}\left(x_{2}, y_{1}\right)\right]\left(u_{i-1}\left(x_{1}, y_{1}\right)\right)-\left(u_{i}\left(x_{1}, y_{1}\right)\right) \\
= & \frac{k}{2 h} v_{i}^{j}\left(x_{1}, y_{1}\right) \leq \frac{k}{2 h}\left|v_{i}^{j}\left(x_{1}, y_{1}\right)\right| \leq \epsilon_{2},
\end{aligned}
$$

and

$$
\begin{aligned}
r_{(n-1)^{2}-1,(n-1)^{2}}^{j}= & {\left[\left[G_{2}\left(u_{i^{*}}^{j}\left(x_{n-1}, y_{n-1}\right)\right)\right]_{x_{n-2}} v_{i}^{j}\left(x_{n-2}, y_{n-1}\right)+\left[G_{2}\left(u_{i^{*}}^{j}\left(x_{n-1}, y_{n-1}\right)\right)\right]_{x_{n-2}} v_{i}^{j}\left(x_{n-1}, y_{n-2}\right)\right.} \\
& \left.+\left[G_{1}\left(u_{i^{*}}^{j}\left(x_{n-1}, y_{n-1}\right)\right)\right]_{x_{n-2}} v_{i}^{j}\left(x_{n-1}, y_{n-1}\right)\right]\left(u_{i-1}\left(x_{n-1}, y_{n-1}\right)\right)-G_{1}\left(u_{i}\left(x_{n-1}, y_{n-1}\right)\right) \\
= & \frac{-k}{2 h} v_{i}^{j}\left(x_{n-1}, y_{n-1}\right) \leq \frac{k}{2 h}\left|v_{i}^{j}\left(x_{n-1}, y_{n-1}\right)\right| \leq \epsilon_{3} .
\end{aligned}
$$

By the same way, we conclude that the elements of matrix $\mathrm{R}$ are small. Thus, the norm of the matrix $\mathrm{R}$, which is defined by $\sup _{x \in \Re^{n-1}}\left\|R\left(v_{i}^{j}, u_{i^{*}}^{j}\right)\right\|$ is small and bounded.

Now, it remains to show that $G$ is invertible and bounded away from zero. 
The matrix $G$ is positive definite and bounded away from zero, since we can choose k and $h$, to such that the diagonal of the matrix is positive [2]. This completes the proof of part (i).

Proof (ii): as in part (i), consider the following iteration

$$
P\left(u_{i}^{j}\right)\left\{u_{i+1}^{j}-u_{i}^{j}\right\}=\left(P\left(u_{i-1}^{j}\right)-P\left(u_{i}^{j}\right)\right)\left\{u_{i}^{j}\right\} \text {. }
$$

By the same argument as in (i), the right hand side of equation(7), becomes

$W\left(v_{i}^{j}, u_{i^{*}}^{j}\right)\left\{u_{i-1}^{j}-u_{i}^{j}\right\}$,

Where, the matrix $W\left(v_{i}^{j}, u_{i^{*}}^{j}\right)$ is the following:

$$
\left[\begin{array}{ccccc}
0 & w_{1,2}^{j} & 0 & 0 & 0 \\
w_{2,1}^{j} & 0 & w_{2,3}^{j} & 0 & 0 \\
\vdots & \vdots & \ddots & \vdots & \vdots \\
0 & 0 & w_{(n-1)^{2}-1,(n-1)^{2}-2}^{j} & 0 & w_{(n-1)^{2}-1,(n-1)^{2}}^{j} \\
0 & 0 & 0 & w_{(n-1)^{2},(n-1)^{2}-1}^{j} & 0
\end{array}\right] .
$$

Since, $\mathrm{k}$ and $\mathrm{h}$ are both small and $u^{j}$ is bounded [1], then the nonzero elements are sufficiently small. Since,

$$
\begin{aligned}
\begin{aligned}
w_{1,1}^{j}= & {\left[p_{1}\left(u_{i^{*}}^{j}\left(x_{1}, y_{1}\right)\right)\right]_{x 1} u_{i}^{j}\left(x_{1}, y_{1}\right)+\left[p_{2}\left(u_{i^{*}}^{j}\left(x_{1}, y_{1}\right)\right)\right]_{x 1} u_{i}^{j}\left(x_{1}, y_{2}\right)+} \\
& \left.+\left[p_{2}\left(u_{i^{*}}^{j}\left(x_{1}, y_{1}\right)\right)\right]_{x 1} u_{i}^{j}\left(x_{2}, y_{1}\right)\right]\left(u_{i-1}\left(x_{1}, y_{1}\right)\right)-\left(u_{i}\left(x_{1}, y_{1}\right)\right)
\end{aligned} \\
\begin{aligned}
w_{1,2}^{j}= & 0, \quad\left[p_{2}\left(u_{i^{*}}^{j}\left(x_{1}, y_{1}\right)\right)\right]_{x_{2}} u_{i}^{j}\left(x_{1}, y_{1}\right)+\left[p_{1}\left(u_{i^{*}}^{j}\left(x_{1}, y_{1}\right)\right)\right]_{x_{2}} u_{i}^{j}\left(x_{1}, y_{2}\right)+ \\
& \left.+\left[p_{2}\left(u_{i^{*}}^{j}\left(x_{1}, y_{1}\right)\right)\right]_{x_{2}} u_{i}^{j}\left(x_{2}, y_{1}\right)\right]\left(u_{i-1}\left(x_{1}, y_{1}\right)\right)-\left(u_{i}\left(x_{1}, y_{1}\right)\right)
\end{aligned} \\
=\frac{k}{2 h} u_{i}^{j}\left(x_{1}, y_{2}\right) \\
\leq \frac{k}{2 h}\left|u_{i}^{j}\left(x_{1}, y_{2}\right)\right| \leq \epsilon_{4},
\end{aligned}
$$

and

$$
\begin{aligned}
w_{(n-1)^{2},(n-1)^{2}-1}^{j}= & {\left[\left[p_{2}\left(u_{i^{*}}^{j}\left(x_{n-1}, y_{n-2}\right)\right)\right]_{x_{n-2}} u_{i}^{j}\left(x_{n-2}, y_{n-2}\right)+\left[p_{2}\left(u_{i^{*}}^{j}\left(x_{n-1}, y_{n-2}\right)\right)\right]_{x_{n-2}} u_{i}^{j}\left(x_{n-1}, y_{n-3}\right)\right.} \\
& \left.\left.+\left[p_{1}\left(u_{i^{*}}^{j}\left(x_{n-1}, y_{n-2}\right)\right)\right]_{x_{n-2}} v_{i}^{j}\left(x_{n-1}, y_{n-2}\right)\right]+\left[p_{2}\left(u_{i^{*}}^{j}\left(x_{n-1}, y_{n-2}\right)\right)\right]_{x_{n-2}} v_{i}^{j}\left(x_{n-1}, y_{n-1}\right)\right]^{*} \\
& \quad\left(u_{i-1}\left(x_{n-1}, y_{n-2}\right)\right)-\left(u_{i}\left(x_{n-1}, y_{n-2}\right)\right) \\
= & \frac{-k}{2 h} u_{i}^{j}\left(x_{n-1}, y_{n-2}\right) \\
& \leq \frac{k}{2 h}\left|u_{i}^{j}\left(x_{n-1}, y_{n-2}\right)\right| \leq \epsilon_{5} .
\end{aligned}
$$

Also, the other elements are sufficiently small. Thus, the norm of the matrix $\mathrm{W}$, which is defined by $\sup _{x \in \Re^{n-1}}\left\|W\left(v_{i}^{j}, u_{i^{*}}^{j}\right)\right\|$ is small and bounded.

Now, it remains to show that $P$ is invertible and bounded away from zero.

The matrix $P$ is positive definite and bounded away from zero, since we can choose $\mathrm{k}$ and $h$, to such that the diagonal of the matrix is positive [2]. This completes the proof of part (ii). 


\section{Numerical Experiments}

In this section, we solved numerically the BBM-BBM system by using the implicit finite difference method with homogeneous Dirichlet boundary conditions in [12]. If we consider the initial-value problem for(2) with a given general initial profile $\left(v_{o}(x, y), u_{o}(x, y)\right)$ with homogenous Dirichlet boundary condition, and apply the scheme in section (2) we solve a system in each step. The termination criterion for the inner iteration was: $\max \left|u_{j}^{(k+1)}-u_{j}^{(k)}\right| \leq .01$ and $\max \left|v_{j}^{(k+1)}-v_{j}^{(k)}\right| \leq .01$, $k=0,1,2, \ldots$ and $j \in N$ is the time step ,

We present the results of numerical experiments that we performed in the case of the BBM-BBM system of two dimension, with homogenous Dirchlet boundary conditions. In many real physical situations, the wave is generated by a source which is not necessarily axisymmetric, for example in the 2004 Asian tsunami the waves were generated by a fault line which is about $1200 \mathrm{~km}$ length in a north-south orientation. It is observed that the greatest strength of the tsunami waves was in an east-west direction [14], and the initial data in this sequence of tests be based on

$$
\begin{aligned}
& v(x, y, 0)=5 \alpha^{2} e^{-\alpha^{2 m}\left(\sigma^{m}\left(x-x_{0}\right)^{2 m}+\sigma^{-m}\left(y-y_{0}\right)^{2 m}\right),} \\
& u(x, y, 0)=0,
\end{aligned}
$$

Where, $\alpha=0.1, m=8, \sigma=10$, [14].

The simulations are executed up to time $\mathrm{t}=10 . u$ and $v$ values at $\mathrm{y}=15, \mathrm{t}=8$ with different values of $x$ presented in table 1 . In figure 1, the initial wave profile of $v$ and its surface plot are presented to give a view on the rectangular nature of the initial data. Similar plots are presented with different time in figure 2. The results show that the waves have the same behavior with the results in [14], where the leading wave in the positive and negative $\mathrm{x}$-directions (east-west directions) is much bigger than that in the north-south directions. The waves in the north-south directions are very small. Also, as in [14], the maximum amplitude is 0.05 at $\mathrm{t}=0$, and its decrease when time increases.

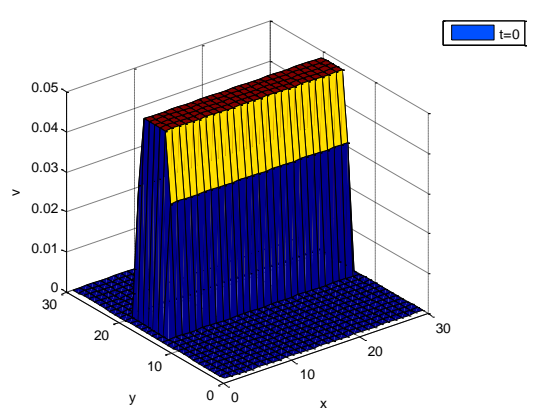

Figure 1. plot of initial data $v(x, y, o)$ 

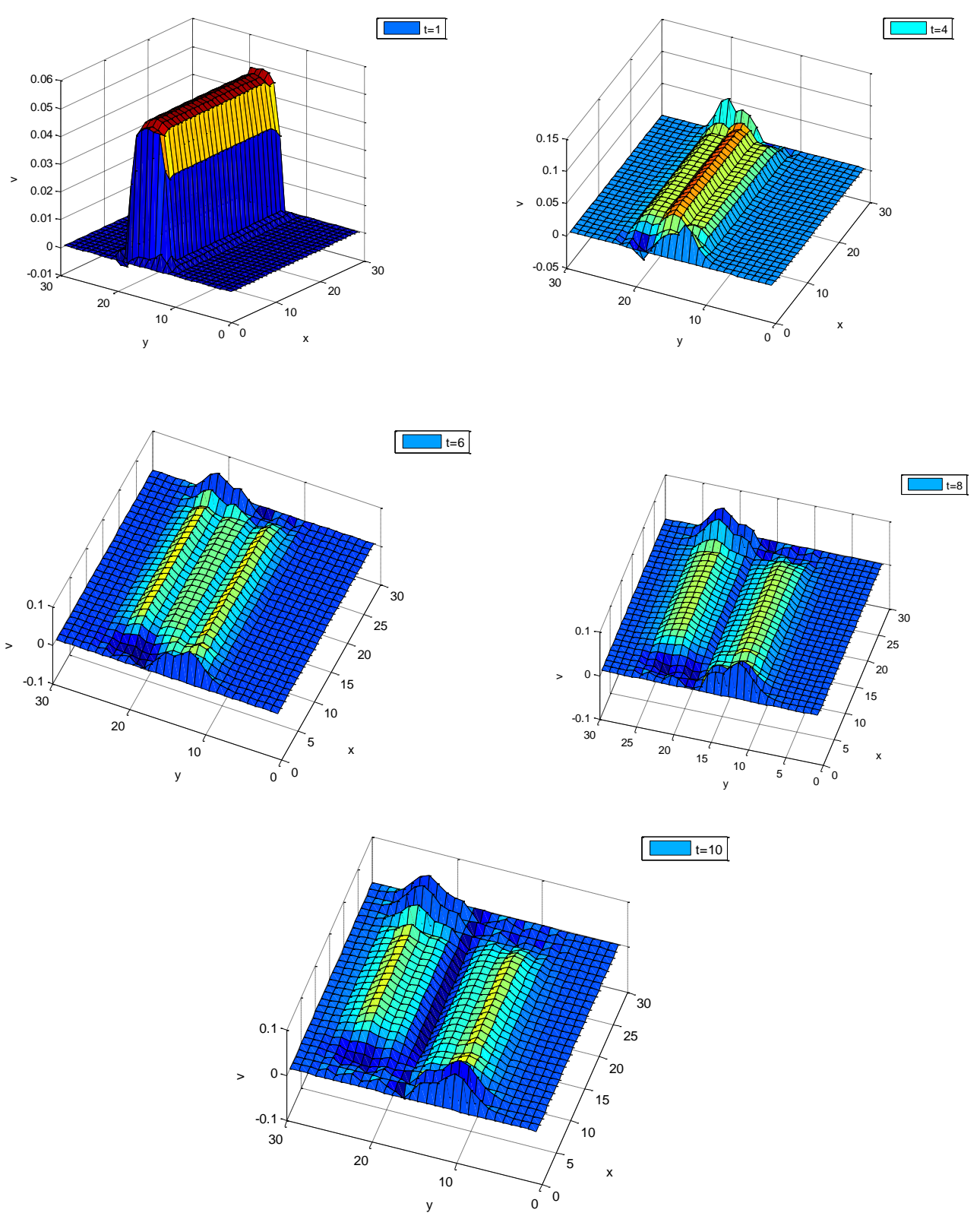

$\mathrm{t}=10$

Figure 2. Solution describes the evolution of $v$ in the system(2) for different time.

Table 1: $u$ and $v$ values at $y=15, \mathrm{t}=8$.

\begin{tabular}{|c|c|c|}
\hline$x$ & $u$ & $v$ \\
\hline 0 & 0 & 0 \\
\hline 1 & 0 & 0 \\
\hline 2 & $-3.8 \mathrm{E}-07$ & $1.37 \mathrm{E}-06$ \\
\hline 3 & $-6.7 \mathrm{E}-06$ & $2.06 \mathrm{E}-06$ \\
\hline 4 & $-1.03 \mathrm{E}-05$ & $3.08 \mathrm{E}-05$ \\
\hline 5 & $-1.27 \mathrm{E}-04$ & $4.73 \mathrm{E}-05$ \\
\hline 6 & $-1.95 \mathrm{E}-04$ & $4.705 \mathrm{E}-04$ \\
\hline 7 & -0.0015 & $7.157 \mathrm{E}-04$ \\
\hline 8 & -0.0023 & 0.0043 \\
\hline 9 & -0.01 & 0.0063 \\
\hline 10 & -0.0142 & 0.0192 \\
\hline 11 & -0.0279 & 0.0256 \\
\hline
\end{tabular}




\begin{tabular}{|c|c|c|}
\hline 12 & -0.0334 & 0.0292 \\
\hline 13 & -0.0221 & 0.0297 \\
\hline 14 & -0.0161 & 0.0194 \\
\hline 15 & -0.0226 & 0.0126 \\
\hline 16 & -0.0191 & 0.01 \\
\hline 17 & $2.077 \mathrm{E}-08$ & $-1.01 \mathrm{E}-04$ \\
\hline 18 & 0.0191 & 0.01 \\
\hline 19 & 0.0226 & 0.0126 \\
\hline 20 & 0.0161 & 0.0194 \\
\hline 21 & 0.0221 & 0.0297 \\
\hline 22 & 0.0334 & 0.0292 \\
\hline 23 & 0.0279 & 0.0256 \\
\hline 24 & 0.0142 & 0.0192 \\
\hline 25 & 0.01 & 0.0063 \\
\hline 26 & 0.0023 & 0.0043 \\
\hline 27 & 0.0015 & $7.157 \mathrm{E}-04$ \\
\hline 28 & $1.94 \mathrm{E}-04$ & $4.692 \mathrm{E}-04$ \\
\hline 29 & $1.34 \mathrm{E}-04$ & $4.945 \mathrm{E}-05$ \\
\hline 30 & 0 & 0 \\
\hline
\end{tabular}

\section{Conclusion}

The basic idea in this paper was to prove that the use of implicit finite difference method in solving two dimensional Coupled BBM system possible and, indeed, been proposed theory in this regard, due to the use of this method had emerged from a system of nonlinear algebraic equations and found the practical part of this paper that the method of the fixed point iteration is to give acceptable results and can be easily used in solving nonlinear system for this type of systems 


\section{REFERENCES}

[1] A.A.Alazman, J.P. Albert, J.L. Bona, M. Chen and J. Wu, Comparison between The BBM equation and a Boussinesq systems, adv. Differential equation, 2(2006), 121-166.

[2] David, Kincaid and W. Cheney, Numerical analysis, Mathematics of Science computing, third edition,(2002), printed in USA.

[3] E.S. Al-Rawi and M.A. Mahmoud, Numerical solution of coupled-BBM systems of Boussinesq type by implicit finite difference method, Iraqi Journal of Statistical Science (20), (2011), 70-82.

[4] H. Chen, M. Chen and N. V. Nguyen, Cnoidal wave solutions to boussinesq systems, 20 (2007), 1443-1461.

[5] J.F. Toland, Existence of symmetric homoclinic orbits for systems of EulerLagrange equations. A. M.S. Proc. Symposia in Pure Mathematics 45 (1986) 447-459.

[6] J. L. Bona and M. Chen, A Boussinesq system for two-way propagation of nonlinear dispersive waves, Physica D 116(1998), 191-224.

[7] J. L. Bona, M. Chen and J.-C. Saut, Boussinesq equations and other systems for small-amplitude long waves in nonlinear dispersive media I: Derivation and the linear theory, J.Nonlinear Sci., 12 (2002), 283-318.

[8] J. L. Bona, M. Chen, J.-C. Saut, Boussinesq equations and other systems for small-amplitude long waves in nonlinear dispersive media II: Nonlinear theory, Nonlinearity, 17 (2004), 925-952.

[9] J.L. Bona, T. Colin and D. Lannes, Long wave approximations for water waves. Arch. Rational Mech. Anal. 178 (2005) 373-410.

[10] K.Omrani and M.Ayadi, Finite difference discretization of the Benjamin-BonaMahony-Burgers equation. Wiley inter science,(2007).

[11] M. Chen, Exact traveling-wave solutions to bi-directional wave equations, International Journal of Theoretical Physics, 37 (1998), 1547-1567.

[12] M. Chen and G. Iooss, Periodic wave patterns of two-dimensional Boussinesq systems, European Journal of Mechanics B Fluids, 25 (2006), 393-405.

[13] M. Chen and G. Iooss , Asymmetrical periodic wave patterns of twodimensional Boussinesq systems, Physica D., 237 (2008), 1539-1552.

[14] M. Chen, Numerical investigation of two-dimensional Boussinesq System, Discrete and continuous Dynamical System, 23(2009) 00.1169-1190.

[15] P.Huang, An analytical technique to solve the BBM-BBM system, Bull.Math.Soc.Sci.Math. Roumani Tome, 54(102)No. 4, (2011), 325-335.

[16] P. J. Lynett, J. C. Borrero, P. L.-F. Liu, C. E. Synolakis, Field survey and numerical simulations: A review of the 1998 Papua New Guinea tsunami, Pure Appl. Geophys. 160 (2003) 2119-2146. 
[17] V. A. Dougalis, D. E. Mitsotakis and J.-C. Saut, On some Boussinesq systems in two space dimensions: theory and numerical analysis, M2AN Math.Model.Numer.Anal. 41(2007), 825-854.

[18] V. A. Dougalis, D. E. Mitsotakis and J.-C. Saut, On initial-boundary value problems for aBoussinesq system of BBM-BBM type in a plane domain, Discrete Contin. Dyn. Syst. 23(2009),1191-1204. 